\title{
Corpus-based Development and Evaluation of a System for Processing Definite Descriptions
}

\author{
Renata Vieira \\ Universidade do Vale do Rio dos Sinos \\ Av. Unisinos 950 - Cx. Postal 275 \\ 93022-000 São Leopoldo RS Brazil. \\ renata@exatas.unisinos.br
}

\author{
Massimo Poesio \\ University of Edinburgh \\ HCRC and Informatics \\ Edinburgh, Scotland \\ Massimo. Poesioded. ac .uk
}

\begin{abstract}
We present an implemented system for processing definite descriptions. The system is based on the results of a corpus analysis previously reported, which showed how common discourse-new descriptions are in newspaper corpora, and identified several problems to be dealt with when developing computational methods for interpreting bridging descriptions. The annotated corpus produced in this earlier work was used to extensively evaluate the proposed techniques for matching definite descriptions with their antecedents, discourse segmentation, recognizing discourse-new descriptions, and suggesting anchors for bridging descriptions.
\end{abstract}

\section{Motivation}

In previous work (Poesio and Vicira, 1998) we reported the results of corpus annotation experiments in which the subjects were asked to classify the uses of definite descriptions in Wall Strec Journal articles according to a scheme derived from work by Hawkins (1978) and Prince (1981) and including three classes: DIREC' $T$ ANAPHORA, DISCOURSENEW, and BRIDGING DESCRIPTION (Clark, 1977). This study showed that about half of the time, definite descriptions are used to introduce a new entity in the discourse, rather than to refer to an object already mentioned. We also observed that our subjects didn't always agree on the classification of a given definite; the problem was especially acute for bridging descriptions.

In this paper, we present an implemented system for processing definite descriptions based on the results of that earlier study. In our system, techniques for recognising discourse-new descriptions play a role as important as techniques for identifying the antecedent of anaphoric ones. The system also incorporates robust techniques for processing bridging descriptions.
A fundamental characteristic of our system is that it was developed so that its performance could be evaluated using the annotated corpus. In the paper, we discuss how we arrived at the optimal version of the system by measuring the performance of each method in this way. Because of the problems observed in our previous study concerning agreement between annotators, we evaluated the system both by measuring precision/recall against a 'gold standard' and by measuring the agreement between the annotation it produces and the annotators.

\section{General Overview}

At the moment, the only systems engaged in semantic interpretation whose performance can be cvaluated on fairly unrestricted text such as the Wall Street Journal articles are based on a shallowprocessing approach, i.e., that do not rely on extensive amounts of hand-coded commonsense knowledge (Carter, 1987; Appelt, 1995; Humphreys et al., 1998).' Our system is of this type: it only relies on structural information, on the information provided by pre-existing lexical sources such as WordNet (Fellbaum, 1998), on minimal amounts of general hand-coded information, and on information that can be acquired automatically from a corpus. Although we believe that quantitative evaluations of the performance of a system on a large number of examples are the only true assessment of its performance, and therefore a shallow processing approach is virtually unavoidable for implemented systems until better sources of commonsense knowledge become available, we do know that this approach limits the performance of a system on those instances of definite descriptions which do require commonsense knowledge for their resolution. (We grouped these in what we call the 'bridging' class.) We

\footnotetext{
'Most systems participating in the Message Understanding Conference (MUC) evaluations are customized to specific domains by adding hand-coded commonsense knowledge.
} 
nevertheless developed heuristic technicues for pro cessing these types of definites als well, which may provide a baseline against which the gains in perfor. mance due to the use of commonsense knowlodge can be assessed more clearly.

Our system attempts to classify each definite description as ciber DIRECT ANAPHORA, DISCOURSL-NLW, and BRIDCHNG I)ISCRIPTION. The first class includes definite descriptions whose head is identical to that of their antecedent, as in a house ... the house. The sceond includes definite descriptions that refer to objects not already mentioned in the rext and not related to any such object. (Some of these definite descriptions refer to objects whose existence is widely known, such as discourse-initial references to the pope; other instances of discourse-new descriptions refer to objects that can be assumed to be unique, even if unfa miliar, such as the first wonan to climb all Scottish Munros.) Iinally, we classify as bridging descriptions all definite descriptions whose resolation de. pends on knowledge of relations between objects, such as definite descriptions that refer to an object related to an entity already introduced in the dis. course by a relation other than identity (Prince's 'infertables'), as in the flat ... the living room; and definite descriptions that refer an object already introduced, but using a different predicate, as in the car ...the vehicle. In addition to this classification, the system tries to identify the antecedents of anaphoric descriptions and the anchors (Fraurud, 1990) of bridging ones. Accordingly, we developed three types of heuristics:

- for resolving directly anaphoric descriptions. These include heuristics for dealing with segmentation and to handle modification.

- for identifying discourse-new descriptions. Some of these heuristics attempt to recognize semantically functional definite descriptions (Hawkins, 1978; Loebner, 1987), whereas othcrs try to recognize definite descriptions that are anchored via their modification (Clark and Marshall, 1981; Prince, 1981).

- for identifying the anchor of a bridging description and the semantic relation between the bridging description and its anchor. WordNet is accessed, and heuristics for named entity recognition were also developed.

The final configuration of the system was arrived at on the basis of an extensive cvaluation of the heuristics using the corpus annotated in our previ. ous work (Poesio and Vicira, 1998). The evaluation was used both to determine which version of each heuristic worked better, and to identify the best ofder in which to try them.

The corpus we used consists of 34 texts from the Penn tredbank 1 included in the $A C I / D C l(D-r o m$. 20 of these texts wore trated as "traming corpus"; this corpus contains 1040 definite descriptions, of which 312 are anaphoric, 492 discourse now, and 204 bridging. 14 more texts were used as 'tost corpus'; these include 464 definite descriptions, of which 154 have been classified as anaphoric, 218 as discourse-new, and 81 as bridging.

\section{The Heuristics And Their Performance}

\subsection{Kesolving Amaphoric Definites}

We discuss heuristies for two subproblems of the task of resolving anaphoric definites: limiting the accessibility of discourse cntitics (segmentation), and taking into account the information given by pre- and post-modifiers. Sec (Vicira, 1998) for a discussion of the other heuristics used by the sys tem.

Segmentation In general, discourse entities have life-spans limited to pragmatically determined $\$$ lic $\mathrm{s}$. MLNTS that may be nested (see, e.g., (Reichman, 1985; Grost and Sidner, 1986; Jox, 1987)). F.g., in our corpus we found that about $10 \%$ of direct anaphoric definite descriptions have more than one possible antecedent if segmentation is not taken into account (Vieira and Poesio, 1999). Recognizing the hierarchical structure of segments in a text is, however, still pretty much an open problem, as it involves reasoning about intentions; ${ }^{2}$ better results have been achieved on the simpler task of 'chunking' the text into approximate segments, generally by means of lexical density measures (Hearst, 1997) - In fact, the methods to limit the lifespan of discourse entity we considered for our system were even simpler. One type of heuristics we looked at are window-based techniques, i.e., considering as potential antecedents only the discourse entitics within fixed-size windows of previous sentences, allowing however for some discourse entities to take a longer life span: we call this method LOOSE SEGmentation. More specifically, a discourse entity is considered as potential antecedent for a definite

\footnotetext{
${ }^{2}$ See, however, (Marcu, 1999).
} 
description when the antecedent's head is identical to the description's head, and

- the potential antecedent's distance from the de scription is within the established window, or elso

0 the potential antecedent is itsell a subsecplent nucilion, or clse

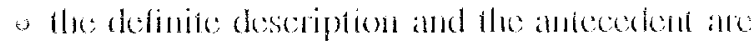
identical NI: (including, the anticle).

Wo also considered an wen simpler RHCWNCY Jemistic: this involves kecping, a table indexed by the heads of potential attocedontes, sten that the aniry for nom $N$ contains the index of the lasi ocente rence of an antecedent will lead N. Jinally, we con sisecel combinations of serimentation and recency.

The best results were obtalined with a combimation of hle recency and sounchation hemistios: just one potential antecedent for anch diflerent heal nom is avabable for resolution, the lasi ocentrence dithat hat roum. The resolution still respects the segmentation hentisice (loose version). The reall (R), precision (ly) and limeasure (li) results for the ivo hentistics atre presented in 'lable 1.3

\begin{tabular}{|c|c|c|c|}
\hline Connbued lacenistics & R & $r^{\prime}$ & f: \\
\hline 4 sentences - reconcy & $7.90(1 \%$ & $8 \% .7 \%$ & $81.4 .1 \%$ \\
\hline is sentences - 1 reconcy & $7 \% .88 \%$ & $34.96 \%$ & $81.27 \%$ \\
\hline
\end{tabular}

Tible 1: Combuning foose seomentation and focency heuristics

The version will higher li value in lable I (4. scrutence window plas recency) was shosen and ased in the lests discussed in the resit of this section. Noun Modifiers In general, when matching a definive description with a potential antecedent the information provided by the prenominal and the postnominal part of the noun plirase also has to be taken in to account: so, for example, a blue car cannot serve as the antecedent for the red car, or the house on the left for the house on the right. 'Jaking propet' care of the semantic contribution of these premodificrs would, in gencral, require commonsense rea-

\footnotetext{
"The standard actinitions of precision and recall from information retrieval were uted: R : mumber of of jects of type $A$ correctly identificel by the system / total number of objects of type $A, P^{\prime}-$ number of correct identifications of objects of type $\Lambda /$ total number of objects of type $\Lambda$ identitied by the system, li: : $\left[: \mathrm{P}^{\prime} / \mathrm{R}+\mathrm{I}\right.$.
}

soning; for the noment, we only developed heuris fic solutions bo the problem, including:

- allowing an antecedent to match with a deli nite description il the premodifiess of the de. scription are a subset of the premodifiess of the antecedent. 'This hentistic deals with def intes which contain less information than the antecedent, such as an old Vichorian housc... the homse, athl prevents matches such as the business commmanity... the younger; moro acaivist black political community.

* allowing a non-premodified attecedent to match with any same head definite. This sice ond hentristic deals with defintes that contain adelitional inlormation, such as a check... the hesi clueck.

The resules of one premodifice mallelime alog. rithm are presented in lable? In that lable we also show the results obtained with a modified matching alogrithm includimg, a third rule, that allows a pres. modified antecedent to match with a dedinite whose ser of pre-modifiers is a supersed of the set of nododi. fics of the andecedent (an elaboration of rule ?). Wo rested each of these thee heuristies alone and their combinations. (Tho fombla line simply repeats the results shown in 'Jilld: 1.)

\begin{tabular}{|c|c|c|c|}
\hline Antecerdents selection & R & l' & $1:$ \\
\hline 1. Ant -sed/I)ese-subsel & (0) $8 \% \%$ & $91.21 \%$ & $79.1 \%$ \\
\hline$\therefore$ Sint-cmlply & $55.12 \%$ & $38.20 \%$ & $6 \% .85 \%$ \\
\hline 3. Ant - subset/1)esc set & $04 \% / 4 \%$ & $88.50 \%$ & $74.81 \%$ \\
\hline I and 2 (basice v) & $75.96 \%$ & $3 \% .71 \%$ & $81.11 \%$ \\
\hline 1 and 3 & $15.96 \%$ & $8 \% .13 \%$ & $81.16 \%$ \\
\hline Nonce & $78.52 \%$ & $81.93 \%$ & $80.10 \%$ \\
\hline
\end{tabular}

'lable 2: livaluation of the lementics for premodifi. cation (version 1)

The best precision is acheved by the matching al. gorithm that does not allow for new information in the allaphoric expression, but the best results overall are again obtained by combining rule 1 and role 2 , although either 2 or 3 works equally well when combined with 1 .

Overall results for anaphoric definite descriptions 'To summarize, the version of the system that achieves the best results as far as anaphoric definito descriptions atre concened includes :

1. combined segmentation and recency, 
2. 4-sentence window,

3. considering indefinites, definites and possessives as potential antecedents (Vieira, 1998),

4. the premodification of the description must be contained in the premodification of the antecedent when the antecedent has no premodifiers.

\subsection{Heuristics for Recognizing Discourse-New Descriptions}

As mentioned above, a central characteristic of our system is that it also includes heuristics for recognising discourse-new descriptions (i.e., definite descriptions that introduce new discourse entities) on the basis of syntactic and lexical features of the noun phrase. Our heuristics are based on the discussion by Hawkins (1978), who identified a number of correlations between certain types of syntactic structure and discourse-new descriptions, particularly those that he called 'unfamiliar' definites (i.e., those whose existence cannot be expected to be known on the basis of generally shared knowledge), including:

- the presence of 'special predicates': 4

- the occurrence of pre-modifiers such as first or best when accompanied with full relatives, e.g., the first person to sail to America (Hawkins calls these 'unexplanatory modifiers'; Loebner (1987) showed how these predicates may license the use of definite descriptions in an account of definite descriptions based on functionality);

- a head noun taking a complement such as the fact that there is life on Earth (Hawkins calls this subclass 'NP' complements');

- the presence of restrictive modification, as in the inequities of the current land-ownership system.

Our system attempts to recognize these syntactic patterns; in addition, it considers as unfamiliar some definites occurring in

\footnotetext{
${ }^{4}$ This list was developed by hand; more recently, Bcan and Riloff (1999) proposed methods for automatically extracting from a corpus such special predicates, i.c., heads that correlate well with discourse novelty.
}

- appositive constructions (e.g., Glenn Cox, the president of Phillips Petroleum Co.);

- copular constructions (e.g.,the man most likely to gain custody of all this is a career politician named David Dinkins).

In our corpus study (Poesio and Vieira, 1998) we found that our subjects did better at identifying discourse-new descriptions all together $(\mathrm{K}=.68)$ than they did at distinguish 'unfamiliar' from 'larger situation' (Hawkins, 1978) cases $(\mathrm{K}=.63)$. This finding was confirmed by our implementation: although each of the heuristics is designed, in principle, to identify only one of the uses (larger situation or unfamiliar), they work better when used all together to the class of discourse new descriptions.

The overall recall and precision results for the heuristics for identifying discourse new descriptions are shown in Table 3. In this Table we do not distinguish between the two types of discourse-new descriptions, 'unfamiliar' and 'larger-situation'. The column headed by (\#) represents the number of cases of descriptions classified as discourse new in the standard annotation; + indicates the total number of discourse-new descriptions correctly identified; - the number of errors. These results are for the version of the system (version 1) that uses the best version of the heuristics for dealing with anaphoric descriptions discussed above, and that doesn't attempt to resolve bridging descriptions .

\begin{tabular}{|l|l|l|l|l|l|l|}
\hline Discourse new & $\#$ & + & - & $\mathrm{R}$ & $\mathrm{P}$ & $\mathrm{F}$ \\
\hline Training data & 492 & 368 & 60 & $75 \%$ & $86 \%$ & $80 \%$ \\
\hline Test data & 218 & 151 & 58 & $69 \%$ & $72 \%$ & $70 \%$ \\
\hline
\end{tabular}

Table 3: Evaluation of the heuristics for identifying discourse new descriptions

\subsection{Bridging Descriptions}

Bridging descriptions are the class of definite descriptions which a shallow processing system is least equipped to handle, and therefore the most crucial indicator of where commonsense knowledge is actually needed. We knew from the start that in general, a system can only resolve certain types of bridging descriptions when supplied with an adequate knowledge base; in fact, the typical way of implementing a system for resolving bridging references has been to restrict the domain and feed the system with hand-coded world knowledge (see, e.g., (Sidner, 1979) and especially (Carter, 1987)). 
Furthermore, the relation between bridging descriptions and their anchors may be arbitrarily complex (Clark, 1977; Sidner, 1979; Prince, 1981; Strand, 1996) and our own results indicate that the same description may relate to different anchors in a text, which makes it difficult to decide what the intended anchor and the intended link are (Poesio and Vieira, 1998). Nevertheless, we fecl that trying to process these definite descriptions is the only way to discover which types of commonsense knowledge are actually needed. .

We began by developing a classification of bridging descriptions according to the kind of information needed to resolve them, rather than on the basis of the possible relations between descriptions and their anchors as usually done in the literature (Vieira, 1998). This allowed us to get an idea of what types of bridging descriptions our system might be able to resolve. We classificd definite descriptions as follows:

- cases based on well-defined lexical relations, such as synonymy, hypernymy and meronymy, that can be found in a lexical database such as WordNet (Fellbaum, 1998)-as in the flat ... the living room;

- bridging descriptions in which the antecedent is a proper name and the description a common noun, whose resolution requires some way of recognizing the type of object denoted by the proper name (as in Bach ... the composer);

- cases in which the anchor is not the head noun but a noun modifying an antecedent, as in the company has been selling discount packages ... the discounts

- cases in which the antecedent (anchor) is not introduced by an NP but by a VP, as in Kadane oil is currently drilling two oil wells. The activity...

- descriptions whose the antecedent is not explicitly mentioned in the text, but is implicitly available because it is a discourse topic-e.g., the industry in a text referring to oil companies;

- cases in which the relation with the anchor is based on more general commonsense knowledge, c.g., about cause-consequence relations.

We developed heuristics for handling the first three of these classes: lexical bridges, bridges based on names, and bridges to entities introduced by nonhead nouns in a compound nominal. We refer the reader to (Vicira, 1998) for discussion of the heuristics for this last class.

Our system attempts to resolve lexical bridges by consulting WordNet to determine if there is a semantic relation between the head noun of the description and the head noun of one of the NPs in the previous five sentences. The results of this search for our training corpus, in which 204 descriptions are classified as bridging, are shown in Table 4 . It is interesting to note that the semantic relations found in this automatic search were not always those observed in our manual analysis.

\begin{tabular}{|l|l|l|l|}
\hline $\begin{array}{l}\text { Bridging } \\
\text { Class }\end{array}$ & $\begin{array}{l}\text { Relations } \\
\text { Found }\end{array}$ & $\begin{array}{l}\text { Right } \\
\text { Anchors }\end{array}$ & $\begin{array}{l}\% \\
\text { Right }\end{array}$ \\
\hline \hline Synonimy & 11 & 4 & $36 \%$ \\
\hline Hyponimy & 59 & 18 & $30 \%$ \\
\hline Meronimy & 6 & 2 & $33 \%$ \\
\hline Sister & 30 & 6 & $20 \%$ \\
\hline Total & 106 & 30 & $28 \%$ \\
\hline
\end{tabular}

Table 4: Evaluation of the search for anchors using WordNet

We developed a simple heuristic method for assigning types to named entities. Our method identified entity types for $66 \%(535 / 814)$ of all names in the corpus (organizations, persons and locations). The precision was $95 \%$. We could have had a better recall if we had adopted more comprehensive lists of cue words, or consulted dictionaries of names as done for the systems participating in MUC-6. There, recall in the named entity task varies from $82 \%$ to $96 \%$, and precision from $89 \%$ to $97 \% .5$

\section{Overall Evaluation of the System}

The order of application of heuristics is as important as the heuristics themselves. The final order of application was also arrived at on the basis of an extensive evaluation (Vieira, 1998), and is based on the following strategy: ${ }^{6}$

\footnotetext{
${ }^{5} \mathrm{~A}$ more recent version of the system using the named entity recognition software developed by HCRC for the MUC-7 competition (Mikheev et al., 1999) is discussed in (Ishikawa, 1998).

${ }^{6}$ We also attempted to learn the best order of application of the heuristics automatically by means of decision tree learning algorithms (Quinlan, 1993), without however observing a significant difference in performance. See (Vicira, 1998) for details.
} 
1. eliminate first some non-anaphoric cases using 'safe' heuristics (first two tests);

2. if that fails, try to find a same head antecedent;

3. if that doesn't work either, look for an indication that the description is discourse new

4. only then try to interpret the definite description as a bridge.

Two versions of the system were evaluated. Version 1 only attempts to resolve anaphoric definite descriptions and to recognize discourse-new ones, using the techniques discussed in 3.1 and 3.2. Version 2 of the system also attempts to recognize bridging descriptions and to identify their anchors. (We evaluated this version separately because we expected it to get worse results, given the problems mentioned in 3.3.) Both versions of the system were evaluated using the corpus obtained in (Poesio and Vieira, 1998), which, as discussed earlier, was divided in a training set of 1,040 definite descriptions and a test set of 464 definite descriptions not looked at while developing the heuristics. We evaluated the systems both by means of precision and recall figures, and by measuring the agreement of the system with our annotators by means of the $\mathrm{K}$ measure (Carletta, 1996).

\subsection{Recall and Precision Figures}

Version 1 of the system assigned a classification to 698 out of 1040 definite descriptions in the training corpus; 342 were not classified. Of these 698, 611 were classified correctly, 87 incorrectly. In the test corpus, 324 definites were classified, 140 weren't; 254 definites were classified correctly. Per-class and overall precision and recall figures were as follows:

\begin{tabular}{|c|c|c|c|c|c|c|}
\hline Task & $\#$ & + & - & $\bar{R}$ & $\mathrm{P}$ & $\mathrm{F}$ \\
\hline \multicolumn{7}{|l|}{ Anaphora classification } \\
\hline Training data & 312 & 243 & 27 & $78 \%$ & $90 \%$ & $83 \%$ \\
\hline Test data & 154 & 103 & 12 & $67 \%$ & $90 \%$ & $77 \%$ \\
\hline \multicolumn{7}{|l|}{ Anaphora resolution } \\
\hline Training data & 312 & 23.7 & 33 & $76 \%$ & $88 \%$ & $81 \%$ \\
\hline Test datta & 154 & 96 & 19 & $62 \%$ & $83 \%$ & $71 \%$ \\
\hline \multicolumn{7}{|l|}{ Discourse new } \\
\hline Training data & 442 & 368 & 60 & $\overline{75 \%}$ & $86 \%$ & $80 \%$ \\
\hline Test data & 218 & 151 & 58 & $69 \%$ & $72 \%$ & $70 \%$ \\
\hline \multicolumn{7}{|l|}{ Overall (Classification) } \\
\hline Training Data (total) & 1040 & 611 & 87 & $59 \%$ & $88 \%$ & $70 \%$ \\
\hline Training Data (in domain) & 804 & 611 & 87 & $76 \%$ & $87 \%$ & $81 \%$ \\
\hline Test Data (total) & 464 & 2.54 & 70 & $55 \%$ & $78 \%$ & $64 \%$ \\
\hline Test Data (in domain) & 372 & 254 & 70 & $68 \%$ & $78 \%$ & $73 \%$ \\
\hline
\end{tabular}

Recall was only $59 \%$ on the training data when considering all definite descriptions, but $87 \%$ when considering only the definite descriptions that our system was actually equipped to handle; if the definite descriptions that the system couldn't classify were treated as discourse-new, we would get $\mathrm{R}=\mathrm{P}$ $=70 \%$.

Version 2 of the system couldn't be evaluated automatically, as the suggestions concerning bridging descriptions have to be evaluated by hand. The system correctly classified 264 out of 464 definite descriptions in the test corpus, for a recall of $57 \%$, but also incorrectly resolved 113 definite descriptions, reducing the overall precision to $70 \%$.

\subsection{Agreement with Annotators}

We also evaluated the system by comparing the class it assigned to each definite with all the classes assigned to that definite by the annotators, i.e., without fixing a 'golden standard'. Version 1 of the system finds a classification for 318 out of 464 definite descriptions in the test data. If all the definite descriptions that the system cannot classify are treated as discourse-new, the agreement between system and the three subjects that annotated this corpus on the two classes first mention (= discourse-old) and subsequent mention (= discourse-new or bridges) is $\mathrm{K}=.7$; this should be compared with an agreement of $\mathrm{K}=.77$ between the three annotators themselves. If instead of counting these definite descriptions as discourse-new we simply do not include them in our measure of agreement, then the agreement between the system and the annotators is $\mathrm{K}=.78$, as opposed to $K=.81$ between the annotators.

Version 2 finds a classification for 355 out of 464 definite descriptions; however, its agreement figures are worse. If we count the cases that the system can't classify as discourse-new, the agreement between the system and the three annotators for three classes is $\mathrm{K}=.57$; if we count them as bridges, $\mathrm{K}=.63$; if we just discard those cases, $\mathrm{K}=.63$ again. (By comparison, the agreement among annotators on the three classes was $\mathrm{K}=.68$ overall, $\mathrm{K}=.70$ on just the cases that the system was able to classify.) The cases that the system can't handle are mainly discourse-new descriptions.

\section{Conclusions}

We have presented a domain independent system for definite description interpretation whose development was based on an empirical study of definite description use that included multi-annotator experiments. The main novelty of this system is that it does not only attempt to find an antecedent for a 
definite description; it also uses methods for recognizing discourse-new descriptions, which our previous studies revealed to be the largest class of definite descriptions in our corpus. Because our system only relies on 'shallow' information, it encounters problems when commonsense reasoning is actually needed; on the other hand, it can be tested on any domain without extensive hand-coding.

We believe that ultimately, the real value of such a system will be that it provides a baseline against which to measure the impact of commonsense knowledge, once heuristics such as those we developed for premodifier matching can be replaced by robust methods for commonsense inference. We already started preliminary work on replacing a hand-crafted lexical knowledge base like WordNet with automatically acquired lexical bases (Poesio et al., 1998; Ishikawa, 1998).

\section{Acknowledgments}

We wish to thank Jean Carletta and Janet Hitzeman for helpful comments and suggestions. This work was supported in part by a fellowship from CNPq, Brazil (Renata Vieira), in part by an EPSRC Advanced Research Fellowship (Massimo Poesio).

\section{References}

D. et al.. Appelt. 1995. SRI International FASTUS system MUC-6 test results and analysis. In Proc. of the Sixth Message Understanding Conference, pages 237-248, Columbia, Maryland, November.

D. L. Bean and E. Riloff. 1999. Corpus-based identification of non-anaphoric noun phrases. In Proc. of the 37th ACL, pages 373-380, University of Maryland.

J. Carletta. 1996. Assessing agreement on classification tasks: the kappa statistic. Computational Linguistics, 22(2):249-254.

D. M. Carter. 1987. Interpreting Anaphors in Natural Language Texts. Ellis Horwood, Chichester, UK.

H. H. Clark and C. R. Marshall. 1981. Definite reference and mutual knowledge. In A. Joshi, B. Webber, and I. Sag, editors, Elements of Discourse Understanding. Cambridge University Press, New York.

H. H. Clark. 1977. Inferences in comprehension. In D. Laberge and S. J. Samuels, editors, Basic Process in Reading: Perception and Comprehension. Lawrence Erlbaum.

C. Fellbaum, editor. 1998. WordNet: An electronic lexical database. The MIT Press.

B. A. Fox. 1987. Discourse Structure and Anaphora. Cambridge University Press, Cambridge, UK.

K. Fraurud. 1990. Definiteness and the processing of NPs in natural discourse. Journal of Semantics, 7:395-433.
B. J. Grosz and C. L. Sidner. 1986. Attention, intention, and the structure of discourse. Computational Linguistics, 12(3):175-204.

J. A. Hawkins. 1978. Definiteness and Indefiniteness. Croom Helm, London.

M. A. Hearst. 1997. TextTiling: Segmenting text into multi-paragraph subtopic passages. Computational Linguistics, 23(1):33-64.

K. Humphreys, R. Gaizauskas, S. Azzam, C. Huyck, B. Mitchell, and H. Cunningham Y. Wilks. 1998. University of Sheffield: description of the LaSIE-II System as used for MUC-7. In Proc. of the Seventh Message Understanding Conference (MUC-7).

T. Ishikawa. 1998. Acquisition of associative information and resolution of bridging descriptions. Master's thesis, University of Edinburgh, Department of Linguistics, Edinburgh, Scotland.

S. Loebner. 1987. Natural language and generalised quantifier theory. In P. Gärdenfors, editor, Generalized Quantifiers, pages 93-108. D. Reidel, Dordrecht.

D. Marcu. 1999. A decision-based approach to rhetorical parsing. In Proc. of the 37th ACL, pages 365-372, University of Maryland, June. ACL.

A. Mikheev, M. Moens, and C. Grover. 1999. Named Entity recognition without gazetteers. In Proc. of $E A C L$, Bergen, Norway. EACL.

M. Poesio and R. Vieira. 1998. A corpus-based investigation of definite description use. Computational Linguistics, 24(2):183-216, June.

M. Poesio, S. Schulte im Walde, and C. Brew. 1998. Lexical clustering and definite description interpretation. In Proc. of the AAAI Spring Symposium on Learning for Discourse, pages 82-89, Stanford, CA, March. AAAI.

E. F. Prince. 1981. Toward a taxonomy of given-new information. In P. Cole, editor, Radical Pragmatics, pages 223-256. Academic Press, New York.

J. R. Quinlan. 1993. C4.5: programs for machine learning. Morgan Kaufmann, San Mateo, CA.

R. Reichman. 1985. Getting Computers to Talk Like You and Me. The MIT Press, Cambridge, MA.

C. L. Sidner. 1979. Towards a computational theory of definite anaphora comprehension in English discourse. Ph.D. thesis, MIT.

K. Strand. 1996. A taxonomy of linking relations. Manuscript. A preliminary version presented at the Workshop on Indirect Anaphora, Lancaster University, 1996.

R. Vieira and M. Poesio. 1999. Processing definite descriptions in corpora. In S. Botley and T. McEnery, editors, Corpus-based and Computational Approaches to Discourse Anaphora. John Benjamins.

R. Vieira. 1998. Definite Description Resolution in Unrestricted Texts. Ph.D. thesis, University of Edinburgh, Centre for Cognitive Science, February. 